\title{
A tuneable micro-optofluidic biconvex lens with mathematically predictable focal length
}

\author{
Chaolong Song• Nam-Trung Nguyen • Say-Hwa Tan • Anand Krishna Asundi \\ School of Mechanical and Aerospace Engineering, Nanyang Technological University, 50 \\ Nanyang Avenue, \\ Singapore 639798, Singapore \\ e-mail: mntnguyen@ntu.edu.sg
}

\begin{abstract}
Recent research efforts have been increasingly focused on the development of microlenses. Of great interest are in-plane optofluidic lenses due to the possibility of integrating them directly into a microfluidic network. Furthermore, the tunability of optofluidic lenses makes them more adaptive to lab-on-a-chip (LOC) system for biological and chemical analysis. However, the predictability of the performance of the current optofluidic lenses is limited by the lack of a simple explicit mathematical model. Previously, we proposed a model based on dipole flow theory to calculate the flow field in a circular chamber where the optofluidic lens is formed. However, this method assumes that both the core and cladding liquids have the same viscosity. In this article, hydrodynamic spreading theory is used to model the opening angles of the core stream and the cladding stream at the entrance of the circular chamber. Subsequently, the curvature of the lens interface for each corresponding opening angle of the core stream can be determined. The focal length of the optofluidic lens formed in the circular chamber can be analytically formulated, which can help to determine the position of focal point for a given flow condition. Experiments were carried out to verify this new model. Ray-tracing method was used experimentally and numerically to measure the focal length. The theoretical results agree well with both experimental and numeric results.
\end{abstract}

Keywords: Micro-optofluidics Liquid lens; Index matching Hydrodynamic spreading; Light focusing

\section{Introduction}

Optical lenses play indispensable roles in applications for biological and chemical analysis. Lenses are needed for both detection and actuation such as single molecule detection (Eigen and Rigler 1994; Wenger et al. 2008), and optical manipulation of living cells as well as viruses and bacteria (Ashkin and Dziedzic 1987; Ashkin et al. 1987). Traditionally, optical systems for these applications were established with bulky external devices, whose optical components were fabricated with solid materials, which inherently limit the tunability of the system. One of the challenges of the present-day research in this field is the miniaturization and integration of optical lenses into a lab-on-a-chip (LOC) system. Efforts have been devoted to exploring novel 
materials for the lens and reducing its size. Using soft-lithography and micromachining technologies, liquid-filled lens arrays or polydimethylsiloxane (PDMS) microlens were fabricated with fixed focal length (Jeong et al. 2004; Yu et al. 2009; Son et al. 2009; Hongbin et al. 2008; Camou et al. 2003). Wenger et al. (Wenger et al. 2008) increased the numerical aperture (NA) of a lens system by employing a latex microsphere. However, these lenses with fixed focal length lack tunability. Furthermore, the lens interface is shaped by a solid material, whose smoothness is limited and dependent on the fabrication technology.

Recently, specific efforts have been dedicated to micro-lenses based on air-liquid and liquidliquid interface. These liquid lenses have atomically smooth interfaces and take advantages of surface tension to maintain the curvature of the interface (Ren et al. 2008; Lopez and Hirsa 2008; Grilli et al. 2008). The characteristic laminar flow in microscale was used to define the shape of the lens (Tang et al. 2008; Rosenauer and Vellekoop 2009; Song et al. 2009a). With respect to the light propagation direction relative to the plane of the substrate, which normally carries the test sample, microlenses can be divided into two categories: in-plane and out-of-plane types. Inplane lenses manipulate light in the plane of the substrate. Out-of-plane lenses focus incident light perpendicular to the plane of the substrate (Tang et al. 2008). The advantage of in-plane lenses over out-of-plane lenses is the possibility of integrating them into microfluidic networks which perform the sample analysis (Shi et al. 2009). A high level of integration furthermore helps to avoid the manual alignment of optical components as well as to reduce the cost of external bulky components.

Different configurations of in-plane light focusing microlenses have been reported. A cylindrical fluidic lens was formed in a two-fluid flow microfluidic system by driving the flow through a $90^{\circ}$ curved microchannel (Mao et al. 2007). Tang et al. (2008) developed a reconfigurable optofluidic lens using liquid-core/liquid-cladding $\left(\mathrm{L}^{2}\right)$ structure. Combining the 90-degree curved microchannel and a $\mathrm{L}^{2}$-based structure, Rosenauer and Vellekoop (2009) constructed an optofluidic lens which can perform three-dimensional (3D) focusing of light. Other concepts, such as creating a refractive index gradient (Mao et al. 2009) and liquid-air interface maintained by active pressure control (Shi et al. 2009; Dong and Jiang 2009), were demonstrated to have a focusing effect on a in-plane light beam. However, the behaviors of these in-plane optofluidic lenses are not fully predictable due to some drawbacks. For example, the curvature of the lens interface formed by introducing a 90-degree curved channel cannot be predefined by an explicit mathematical formula. The shape of its interface is not spherical and can cause severe optical aberration. The same situation happens with the $\mathrm{L}^{2}$ optofluidic lens developed in a rectangular chamber (Tang et al. 2008), especially when the lens interface approaches the straight side-wall of chamber leading to a flat paraxial region. This flatness depends on the streamlines of flow field inside the chamber which in turn depends on the flow rate ratio, the viscosity ratio, and the density ratio between the core and cladding liquids. All these factors make the focal length and beam quality unpredictable without a simple explicit model.

Previously, we reported the modeling and characterization of an optofluidic lens with a perfect arc shape formed in a circular chamber (Song et al. 2009b). The curvature of the lens interface has a mathematical relationship with the flow rate ratio between core and cladding streams. Therefore, the focal length of the optofluidic lens can be well predicted before the design of an adaptive optical system. However, this theory assumes that the core liquid and the cladding liquid have the same viscosity. Normally, the lens requires the core and cladding liquids to have different refractive indices, and thus also different viscosities. In this article, we introduce a new 
analytic model based on hydrodynamic spreading theory (Nguyen et al. 2007, 2008; Wu and Nguyen 2005) to address the problem of mismatched viscosities of the core liquid and the cladding liquid. Experiments were carried out to verify our new model. Furthermore, ray-tracing method was applied experimentally and numerically to demonstrate that our lens has a mathematically predefined focal length. This mathematical predictability of our microoptofluidic lens could help us to simplify the overall design of optical components in a LOC system.

\section{Theoretical analysis}

In the past, liquid-core liquid-cladding $\left(\mathrm{L}^{2}\right)$ structures have been employed to configure microoptofluidic components such as waveguide (Nguyen et al. 2007; Wolfe et al. 2004), lens (Tang et al. 2008; Song et al. 2009b), and prism (Song et al. 2010). Figure 1a shows the model of $\mathrm{L}^{2}$ system for optical splitter and switch based on hydrodynamic spreading theory (Nguyen et al. 2007, 2008; Wu and Nguyen 2005). Assuming that the three streams comprise the liquids with different viscosities and flow rates, and the viscosity ratios and flow rate ratios are

$\beta_{1}=\mu_{1} / \mu_{0}, \quad \beta_{2}=\mu_{2} / \mu_{0}$

and

$\phi_{1}=Q_{1} / Q_{0}, \quad \phi_{2}=Q_{2} / Q_{0}$

respectively. In the above equations, $\mu$ and $\mathrm{Q}$ represent the viscosity and flow rate, respectively. The core stream and the two cladding streams are denoted with the subscripts 0 , 1 , and 2 , respectively. Based on the model of hydrodynamic focusing reported previously in Nguyen et al. (2007), the width ratios can be estimated as

$W_{0} / W_{1}=1 /\left(1+2 \beta_{1} \phi_{1}\right), \quad W_{0} / W_{2}=1 /\left(1+2 \beta_{2} \phi_{2}\right)$.

Considering a symmetric system (the two cladding streams of the same liquid have the same flow rate), the relationship between the flow rate ratio $\left(\mathrm{Q}_{\text {core }} / \mathrm{Q}_{\text {cladding }}\right)$ and widths of the core and cladding streams can be derived from Eq. 3:

$\beta \cdot \frac{Q_{\text {core }}}{Q_{\text {cladding }}}=\frac{2 W_{0}}{W_{1}-W_{0}}$

where $\beta=\mu_{\text {core }} / \mu_{\text {cladding }}$ is the viscosity ratio between cladding and core liquid. In this configuration as shown in Fig. 1a, all the streamlines are parallel to the side wall of the channel because of the characteristic laminar flow in microscale.

Fig. 1b shows another situation of hydrodynamic spreading. The streamlines spread from a source point A and flow back to a sink point B in a circularly bounded domain. All streamlines inside the circular domain have an arc-shape (Song et al. 2009b). At the beginning of the streamlines (source point $\mathrm{A}$ ), the flow rate ratio as well as the viscosity ratio conjunctly decide the opening angles of the core stream and the cladding stream. Consequently, the curvature of the interface between the core stream and the cladding stream can be determined, since each 
streamline path has its unique arc-shape in a circular chamber. Equation 4 can be written in the angular form as

$\beta \cdot \frac{Q_{\text {core }}}{Q_{\text {cladding }}}=\frac{2 \theta}{\pi / 2-\theta}$

where $2 \theta$ and the $\pi / 2-\theta$ are the opening angles of the core stream and the cladding stream at the source point $\mathrm{A}$, respectively. Equation 5 also assumes that the two cladding streams are symmetric. Since $\overline{\mathrm{AM}}$ is the tangent of the arc $\widehat{\mathrm{APB}}$; the angle $\angle \mathrm{PAO}$ is derived from geometric rules as

$\angle \mathrm{PAO}=\frac{\theta}{2}=\arctan (y / R)$

where $\mathrm{O}$ is the center of the circular domain, $\mathrm{P}$ is the middle point of $\widehat{\mathrm{APB}}$; $\mathrm{y}$ is the length of $\overline{\mathrm{PO}}$; and $\mathrm{R}$ is the radius of the circular domain. The length $\mathrm{y}$ can be represented by a function of the radius $r$ of the curvature $\widehat{A P B}$ as

$y=r-\sqrt{r^{2}-R^{2}}$

By substituting (6) and (7) into (5), the relationship between flow rate ratio and the radius of the interface can be analytically described as

$\frac{Q_{\text {core }}}{Q_{\text {cladding }}}=\frac{1}{\beta} \cdot \frac{2 \arctan \left(r / R-\sqrt{r^{2}-R^{2}} / R\right)}{\pi / 4-\arctan \left(r / R-\sqrt{r^{2}-R^{2}} / R\right)}$

If the core liquid and cladding liquid have the same viscosity, then Eq. 8 reduces to the same form previously derived by the model based on the theory of dipole flow bounded in a circular domain (Song et al. 2009b).

In the case of a thin symmetric biconvex lens, the focal length can be formulated as

$f=\frac{r}{2(n-1)}$

where $r$ is the radius of the interface curvature, and $n$ is the relative refractive index of the core stream. Substituting (9) into (8) leads to the relationship between the flow rate ratio and the focal length:

$\frac{Q_{\text {core }}}{Q_{\text {cladding }}}=\frac{1}{\beta} \cdot \frac{2 \arctan \left(\frac{2(n-1) f-\sqrt{4(n-1)^{2} f^{2}-R^{2}}}{R}\right)}{\pi / 4-\arctan \left(\frac{2(n-1) f-\sqrt{4(n-1)^{2} f^{2}-R^{2}}}{R}\right)}$

Considering the asymmetric case where the two cladding streams of the same liquid have different flow rates, the flow rate of cladding 1 (Fig. 1c) is equal to that of the core stream. By 
varying the flow rate ratio, asymmetric lenses can be formed, and the radii of the upper and lower interface can be derived in the same way as shown in Eqs. 1-3:

$$
\begin{aligned}
\frac{Q_{\text {cladding 2 }}}{Q_{\text {core }}}=\frac{Q_{\text {cladding 2 }}}{Q_{\text {cladding 1 }}}= & \frac{(1+\beta)\left(2 \arctan \left(r_{1} / R-\sqrt{r_{1}^{2}-R^{2}} / R\right)+\pi / 2\right)-\pi}{\beta\left(\pi / 2-2 \arctan \left(r_{1} / R-\sqrt{r_{1}^{2}-R^{2}} / R\right)\right)} \\
& =\frac{(1+\beta)\left(\arctan \left(r_{2} / R-\sqrt{r_{2}^{2}-R^{2}} / R\right)+\pi / 4\right)}{\beta\left(\pi / 4-\arctan \left(r_{2} / R-\sqrt{r_{2}^{2}-R^{2}} / R\right)\right)}
\end{aligned}
$$

where $r_{1}$ and $r_{2}$ are the radii of interfaces $\widehat{\mathrm{APB}}$ and $\widehat{\mathrm{AQB}}$, respectively (Fig. 1c). When the core and cladding streams are of same liquid $(\beta=1)$, Eq. 11 can be simplified into the same form as reported in our previous study (Song et al.2009b).

\section{Experimental setup}

Figure $2 \mathrm{a}$ depicts the schematic configuration of a liquid-core liquid-cladding $\left(\mathrm{L}^{2}\right)$ optofluidic biconvex lens developed in a circular chamber. When the core liquid sandwiched by two cladding liquids enters the circular chamber, it follows the arc-shape streamlines inside the chamber and, therefore, forms the typical shape of a biconvex lens. In our experiment, cinnamaldehyde (Sigma-Aldrich, USA) with a viscosity of $\mu=5.7 \times 10^{-3} \mathrm{Ns} / \mathrm{m}^{2}$ at $25^{\circ} \mathrm{C}$ and a refractive index of $n=1.62$ serves as the core stream to shape the lens. A mixture of $73.5 \%$ ethylene glycol and $26.5 \%$ ethanol by weight with a viscosity of $\mu=9.8 \times 10^{-3} \mathrm{Ns} / \mathrm{m}^{2}$ at $25^{\circ} \mathrm{C}$ and refractive index of $\mathrm{n}=1.412$ matching that of polydimethylsioxane (PDMS) is used as the cladding stream. The curved interface and the mismatched refractive indices between the core stream and the cladding stream allow this configuration to focus in-plane light. By adjusting the flow rate ratio $\left(\mathrm{Q}_{\text {core }} / \mathrm{Q}_{\text {cladding }}\right)$, the interface between core and cladding liquids can be tuned accordingly. As a result, the curvature of the interface as well as the focal length of the lens can be manipulated. In order to further investigate the viscosity mismatch effect, two other liquid systems are used for verifying the model. The first liquid system consists of DI water with a viscosity of $\mu=1.0 \times 10^{-3} \mathrm{Ns} / \mathrm{m}^{2}$ as the core, and the mixture of $55 \%$ glycerol and $45 \%$ DI water with a viscosity of $\mu=7.6 \times 10^{-3} \mathrm{Ns} / \mathrm{m}^{2}$ as the cladding. The second liquid system comprises the mixture of $20 \%$ glycerol and $80 \%$ DI water with a viscosity of $\mu=1.8 \times 10^{-3} \mathrm{Ns} / \mathrm{m}^{2}$ as the core, and DI water as the cladding.

The device was fabricated in PDMS using standard soft lithography technique. The lens design including the microchannel network was printed on a transparency film with a resolution of $8,000 \mathrm{dpi}$. The transparency mask was subsequently used for defining the negative mold of the lens in a 150-lm thick SU-8 layer, which is shown in Fig. 3. The images were taken using scanning electron microscopy (SEM). PDMS was mixed in a weight ratio of 10:1 and poured onto the SU-8 mold working as a master. Subsequently, the PDMS was placed in vacuum for $2 \mathrm{~h}$ to remove the gas bubbles. After the degassing process, the PDMS was heated at $80^{\circ} \mathrm{C}$ for $2 \mathrm{~h}$. The PDMS piece was then peeled off from the master mould, and $0.75-\mathrm{mm}$ diameter access holes were punched using the Harris unicore puncher. The PDMS with the lens structure was subsequently bonded to another flat PDMS part after treating both surfaces with oxygen plasma. The bonded PDMS was then placed in an oven at $150^{\circ} \mathrm{C}$ for $2 \mathrm{~h}$ to ensure good bonding between both surfaces. Needles with an inner diameter of $0.33 \mathrm{~mm}$ and an outer diameter of $0.64 \mathrm{~mm}$ were press fit into the access holes and worked as fluidic interconnects. Applying this 
technology, a circular lens chamber, as well as the microfluidic network with height of $150 \mathrm{~lm}$ was realized.

A multimode optical fiber (AFS105/125Y, THORLABS Inc.) with a numerical aperture of NA $=0.22$ was inserted into a pre-fabricated microchannel with a width of $130 \mu \mathrm{m}$ to work approximately as a point light source. The optical fiber was located at a distance of $4 \mathrm{~mm}$ from the center of the circular chamber which has a diameter of $1 \mathrm{~mm}$. An aperture was formed by filling black ink into the two channels with a width of $50 \mu \mathrm{m}$. The size of the aperture is $350 \mu \mathrm{m}$. The purpose of the aperture is to confine the divergence of the light beam, and to allow only the paraxial rays to incident on the optofluidic lens. Therefore, the spherical aberration can be reduced and the quality of the focused light beam can be improved. Between the optical fiber and the aperture stands a hollow chamber for positioning the tip of the optical fiber. Before the experiment, a mixture of $73.5 \%$ ethylene glycol and $26.5 \%$ ethanol was injected into this hollow chamber to form an index-matching medium between the optical fiber and the lens chamber. In order to visualize the light beam focused by the lens, a ray-tracing chamber was placed behind the circular chamber along the light propagation direction.

Fluorescence dye Rhodamine B (Sigma-Aldrich, excitation wavelength of $540 \mathrm{~nm}$, emission wave length of $625 \mathrm{~nm})$ diluted in a mixture of glycerol $(60 \%$ by weight) and water (40\% by weight) which has a matching refractive index of $n=1.412$ was injected into the ray-tracing chamber to visualize the light rays. The bright-colored field images were recorded with a digital camera (DCRDVD803E, SONY), while the gray-scale fluorescent images were recorded with a interline transfer CCD camera (Sony ICX 084).

\section{Results and discussion}

In order to verify the new model based on hydrodynamic spreading for predicting the relationship between the flow rate ratio and the curvature of the interface, the liquids kept in 5$\mathrm{ml}$ glass syringes were driven by two syringe pumps (KDS230, KD Scientific Inc, USA) into the PDMS chip. An inverted microscope (Nikon Eclipse TE 2000-S, Japan) was used to capture the images. In the experiment, we fixed the flow rates of the two cladding streams at $1 \mathrm{ml} / \mathrm{h}$, and varied the flow rate of the core stream from 1 to $13 \mathrm{ml} / \mathrm{h}$. By increasing the flow rate of the core stream, the opening angle of the core at the entrance of the circular chamber can be enlarged. Since all the streamlines inside a circular chamber follow arc-shape paths, the expansion of the opening angle of the core stream makes the interface between core and cladding streams more curved. There-fore, the curvature of the interface increases with increasing flow rate ratio $\left(\mathrm{Q}_{\text {core }} / \mathrm{Q}_{\text {cladding }}\right)$, as shown in Fig. 3. Owing to the geometric advantage of the circular-shaped boundary, the curvature of the interface does not decrease when the interface approaches the side wall of the chamber, but gets closer to 1 , which is the normalized curvature of the boundary of the circular chamber. This trend was also observed previously using the same liquid for core and cladding streams (Song et al. 2009b). The experimental results agree well with the theoretical analysis through the whole tuning range (Fig. 4) indicating that our theory can be properly applied to the optical design of a lens with a specific curvature of the interface. Furthermore, the test of different liquid systems also validates that this theory can be used to address the problem of mismatched viscosities.

Ray-tracing method was carried out experimentally and numerically to verify the analytic model. A green laser with a wavelength of $\lambda=532 \mathrm{~nm}$ was used to excite the fluorescence dye in the ray-tracing chamber and, therefore, experimentally trace the light rays refracted by the opto- 
fluidic biconvex lens. Numeric simulation was carried out with a program written in MATLAB (MathWorks, Inc.). In the simulation, light was assumed to emit from a point source, and the refraction at each interface during the propagation of light was calculated using Snell's law. The flow rate ratio $\left(\mathrm{Q}_{\text {core }} / \mathrm{Q}_{\text {cladding }}\right)$. The interface involved in the simulation is the theoretical arc calculated from the corresponding flow rate ratios used in the experiments. Figure 5 compares the experimental with numeric results. These results were obtained at the core stream (cinnamaldehyde with a refractive index of $n=1.62$ ) flow rates of $1,5,9,13$, and $17 \mathrm{ml} / \mathrm{h}$, while keeping the two cladding stream (a mixture of $73.5 \%$ ethylene glycol and $26.5 \%$ ethanol with a refractive index of $\mathrm{n}=1.412$ ) at a fixed flow rate of $1 \mathrm{ml} / \mathrm{h}$. By increasing the flow rate of the core stream in the experiment, the light beam was manipulated from being slightly divergent to well focused. It can be found that even when the flow rate ratio $\left(\mathrm{Q}_{\text {core }} / \mathrm{Q}_{\text {cladding }}\right)$, is increased to a very large value $(\phi=17)$, the image distance does not elongate but keeps on shortening. This is different from the previous case of using a square chamber to develop the optofluidic lens (Tang et al. 2008). In that previous case, the paraxial region of the lens interface becomes flat when the flow rate ratio $\left(\mathrm{Q}_{\text {core }} / \mathrm{Q}_{\text {cladding }}\right)$ reaches a high value due to the geometric limit. Therefore, the focal length will elongate in an unpredictable way.

Figure $6 \mathrm{a}$ shows the ray-tracing image in gray scale, and the corresponding result of quantitative analysis is shown in Fig. 6b. The light beam was focused under the condition of 13 $\mathrm{ml} / \mathrm{h}$ core stream and $1 \mathrm{ml} / \mathrm{h}$ cladding streams. The intensity profiles at different positions (Fig. 6a) were extracted and fitted with Gaussian curves. All the intensity values were normalized by the maximum value of intensity along line A. The intensity distributions at 870, 3,100, and 3,600 $\mu \mathrm{m}$ away from the center of the circular chamber were investigated. We define the full width at half maximum (FWHM) of the fitting curve as the width of light beam. The beam widths were measured as 185,52 , and $74 \mu \mathrm{m}$ for line $\mathrm{C}$, A, and B, respectively. From this quantitative analysis, we found that the light beam becomes convergent after being refracted by the lens, and propagated divergently after the focus.

For the measurement of focal lengths under different flow rate ratios $\left(\mathrm{Q}_{\text {core }} / \mathrm{Q}_{\text {cladding }}\right)$, we fixed the flow rates of two cladding stream (a mixture of $73.5 \%$ ethylene glycol and $26.5 \%$ ethanol with a refractive index of $\mathrm{n}=1.412$ ) at $1 \mathrm{ml} / \mathrm{h}$, and varied the flow rate of core stream (cinnamaldehyde with a refractive index of $\mathrm{n}=1.62$ ) from 2 to $18 \mathrm{ml} / \mathrm{h}$. At each flow rate ratio condition, we captured the gray-scale image of the light rays, and extracted the ray with the angular aperture in the image space, and calculated the intersection position of the ray and the optical axis, by which the image distance can be retrieved. By applying the conjugate relationship between the object and the image, the focal length of the optofluidic lens for each corresponding flow rate ratio can be obtained. The experimental measurement of focal length is shown as the circles in Fig. 7. The solid line in Fig. 7 represents the curve of Eq. 10. The cross marks denote the results computed by numeric simulation of the ray propagation. The experimental results agree well with the analytic analysis as well as the numeric simulation. Therefore, our theoretical model allows for the design of an optofluidic lens with a specific focal length by properly choosing a corresponding flow rate ratio. For reference, the inset in Fig. 7 shows the axial aberration $(\delta \mathrm{L})$ retrieved from numeric ray tracing.

An interesting observation during our experiment was the light scattering at the chamber wall. If the circular chamber is fully filled with cinnamaldehyde $(n=1.62)$, then the light rays behind the optofluidic lens becomes scattered and not sharply focused, Fig. 8(b). If the chamber is filled with the mixture of ethylene glycol and ethanol $(\mathrm{n}=1.412)$, then the light rays propagate without refraction or scattering, Fig. 8a. This two cases reveal that the mismatch of refractive indices 
between cinnamaldehyde ( $\mathrm{n}=1.62)$ and PDMS $(\mathrm{n}=1.412)$ and the surface roughness of the lens chamber (Fig. 3) cause the diffusive reflection and refraction of light rays at the liquid-solid interface leading to strong scattering. This phenomenon confirms the significance of cladding liquid with a refractive index matched to that of PDMS. Besides the roles of shaping the lens and forming an atomically smooth interface, a cladding liquid with a matched refractive index reduces scattering of light at the liquid-solid interface and thus improves the beam quality.

\section{Conclusions}

A liquid-core/liquid-cladding optofluidic lens has a tunable focal length by adjusting the flow rate ratio between core stream and cladding stream. These lenses can perform in-plane light focusing, which can help us to avoid manual optical alignment and bulky external optical components. With this technology, the level of integration of the whole system can be enhanced leading to reduced cost. The challenging problem is to design such a lens system with a mathematically predictable focal length. Previously, we proposed to use dipole flow theory to model the flow field in a circular chamber where the optofluidic lens is developed, and calculate the focal length. However, this method is limited by the assumption that both core and cladding liquids have the same viscosity (Song et al. 2009b). In this article, we employ the theory of hydrodynamic spreading to calculate the opening angles of core and cladding streams at the entrance of the circular chamber and there-after retrieve the corresponding curvature of the interface for each flow rate ratio. Combining with Gaussian optics model, the focal length of the liquid-core liquid-cladding optofluidic lens can be mathematically formulated. Experiments were carried out to validate our theory. We used the ray-tracing method experimentally and numerically to measure the focal length of the optofluidic biconcave lens. The good agreement between our theoretical, experimental, and numeric results shows that the model presented in this article can be used to design a microlens system with a mathematically predefined focal length for each lens. This can help us to simplify the overall design of optical components in a LOC system. 


\section{References}

[1] Ashkin A, Dziedzic JM (1987) Optical trapping and manipulation of viruses and bacteria. Science 235:1517-1520

[2] Ashkin A, Dziedzic JM, Yamane T (1987) Optical trapping and manipulation of single cells using infrared laser beams. Nature 330:769-771

[3] Camou S, Fujita H, Fujii T (2003) PDMS 2D optical lens integrated with microfluidic channels: principle and characterization. Lab Chip 3:40-45

[4] Dong L, Jiang H (2009) Tunable and movable liquid microlens in situ fabricated within microfluidic channels. Appl Phys Lett 91:041109

[5] Eigen M, Rigler R (1994) Sorting single molecules: application to diagnostics and evolutionary biotechnology. Proc Natl Acad Sci USA 91:5740-5747

[6] Grilli S, Miccio L, Vespini V, Finizio A, De Nicola S, Ferraro P (2008) Liquid micro-lens array activated by selective electrowetting on lithium niobate substrates. Opt Express 16:80848093

[7] Hongbin Y, Guangya Z, Siong CF, Feiwen L (2008) Simple method for fabricating solid microlenses with different focal lengths. IEEE Photonics Technol Lett 20:1624-1626

[8] Jeong KH, Liu GL, Chronis N, Lee LP (2004) Tunable microdoublet lens array. Opt Express 12:2494-2500

[9] Lopez CA, Hirsa AH (2008) Fast focusing using a pinned-contact oscillating liquid lens. Nat Photonics 2:610-613

[10] Mao X, Waldeisen JR, Juluri BK, Huang TJ (2007) Hydrodynamically tunable optofluidic cylindrical microlens. Lab Chip 7:1303-1308

[11] Mao X, Lin SCS, Lapsley MI, Shi J, Juluri BK, Huang TJ (2009) Tunable Liquid Gradient Refractive Index (L-GRIN) lens with two degrees of freedom. Lab Chip 9:2050-2058

[12] Nguyen NT, Kong TF, Goh JH, Low CLN (2007) A micro optofluidic splitter and switch based on hydrodynamic spreading. J Micromech Microeng 17:2169-2174

[13] Nguyen NT, Yap Y, Sumargo A (2008) Microfluidic rheometer based on hydrodynamic focusing. Meas Sci Technol 19:085405

[14] Ren H, Xianyu H, Xu S, Wu ST (2008) Adaptive dielectric liquid lens. Opt Express $16: 14954-14960$

[15] Rosenauer M, Vellekoop MJ (2009) 3D fluidic lens shaping-a multiconvex hydrodynamically adjustable optofluidic microlens. Lab Chip 9:1040-1042 
[16] Shi J, Stratton Z, Lin SCS, Huang H, Huang TJ (2009) Tunable optofluidic microlens through active pressure control of an air- liquid interface. Microfluid Nanofluid 1-6

[17] Son HM, Kim MY, Lee YJ (2009) Tunable-focus liquid lens system controlled by antagonistic winding-type SMA actuator. Opt Express 17:14339-14350

[18] Song C, Nguyen NT, Asundi AK, Low CLN (2009a) Biconcave micro-optofluidic lens with low-refractive-index liquids. Opt Lett 34:3622-3624

[19] Song C, Nguyen NT, Tan SH, Asundi AK (2009b) Modelling and optimization of micro optofluidic lenses. Lab Chip 9:1178-1184

[20] Song C, Nguyen N-T, Asundi AK, Tan S-H (2010) Tunable micro-optofluidic prism based on liquid-core liquid-cladding configuration. Opt Lett 35:327-329

[21] Tang SKY, Stan CA, Whitesides GM (2008) Dynamically reconfig-urable liquid-core liquid-cladding lens in a microfluidic channel. Lab Chip 8:395-401

[22] Wenger J, Gerard D, Aouani H, Rigneault H (2008) Disposable microscope objective lenses for fluorescence correlation spec-troscopy using latex microspheres. Anal Chem 80:6800-6804

[23] Wolfe DB, Conroy RS, Garstecki P, Mayers BT, Fischbach MA, Paul KE, Prentiss M, Whitesides GM (2004) Dynamic control of liquid-core/liquid-cladding optical waveguides. Proc Natl Acad Sci USA 101:12434-12438

[24] Wu Z, Nguyen NT (2005) Hydrodynamic focusing in microchannels under consideration of diffusive dispersion: theories and exper-iments. Sensors Actuators B 107:965-974

[25] Yu HB, Zhou GY, Chau FK, Lee FW, Wang SH, Leung HM (2009) A liquid-filled tunable double-focus microlens. Opt Express 17:4782-4790 


\section{List of Figures}

Fig. 1 Schematic of hydrodynamic spreading in microfluidic channels of different geometries: a straight-line channel; b and c circularly bounded chamber

Fig. 2 Schematic configuration of the experimental setup for focusing the light beam emitted from an optical fiber by an $\mathrm{L}^{2}$ optofluidic biconvex lens developed in a circular chamber. The optical fiber is located at a distance of $4 \mathrm{~mm}$ from the center of the circular chamber with a diameter of $1 \mathrm{~mm}$. An aperture is built by filling black ink into two channels with a width of $50 \mu \mathrm{m}$. The size of aperture is $350 \mu \mathrm{m}$. A hollow rectangular chamber is positioned between the optical fiber and the aperture for later injection of a liquid with a matching refractive index. a A ray-tracing chamber filled with fluorescence dye solution is placed behind the circular chamber to visualize the light propagation. All microfluidic structures have a height of $150 \mu \mathrm{m}$. b Bright field image of the $\mathrm{L}^{2}$ optofluidic biconvex lens focusing a light beam. Cinnamaldehyde is used as the core stream, and a mixture of $73.5 \%$ ethylene glycol and $26.5 \%$ ethanol served as the cladding stream. The flow rate ratio between core and cladding streams is 7

Fig. 3 Scanning electron microscopy of the microstructure of SU-8 mold

Fig. 4 Relationship between the curvature of the interface and the flow rate ratio $\left(Q_{\text {core }} / Q_{\text {cladding }}\right)$. The solid line represents the analytic result. The discrete points denotes the experimental results. The flow rates of two cladding streams are kept the same as $1 \mathrm{ml} / \mathrm{h}$. The flow rate of the core stream varies to achieve symmetrical biconvex lenses with different interface curvatures. Different combination of liquids are used in the test: (1) cinnamaldehyde with a viscosity $\mu=5.7 \times 10^{-3}$ $\mathrm{Ns} / \mathrm{m}^{2}$ as core stream and the mixture of $73.5 \%$ ethylene glycol and $26.5 \%$ ethanol with a viscosity $\mu=9.8 \times 10^{-3} \mathrm{Ns} / \mathrm{m}^{2}$ as cladding stream (viscosity ratio $\left.\mu_{\text {core }} \mu_{\text {cladding }}=0.58\right)$; (2) DI water with a viscosity of $\mu=1.0 \times 10^{-3} \mathrm{Ns} / \mathrm{m}^{2}$ as core stream, and the mixture of $55 \%$ glycerol and $45 \%$ DI water with a viscosity of $\mu=$ 
$7.6 \times 10^{-3} \mathrm{Ns} / \mathrm{m}^{2}$ as cladding (viscosity ratio $\mu_{\text {core }} / \mu_{\text {cladding }}=0.13$ ); (3) the mixture of $20 \%$ glycerol and $80 \%$ DI water with a viscosity of $\mu=1.8 \times 10^{-3} \mathrm{Ns} / \mathrm{m}^{2}$ as core, and DI water as cladding (viscosity ratio $\mu_{\text {core }} \mu_{\text {cladding }}=1.8$ ). The insets illustrate the images of the lens at different flow rate ratios by using cinnamaldehyde as core stream and the mixture of $73.5 \%$ ethylene glycol and $26.5 \%$ ethanol as cladding stream. The dimensions are normalized by the radius of the circular chamber. The error bars show the fluctuation of flow inside the circular chamber

Fig. 5 Comparisons between experimental and numeric ray-tracing under conditions of different flow rate ratios

Fig. 6 The intensity distribution of fluorescence at different locations $(A-C)$ in the raytracing chamber. The light beam was focused by an optofluidic biconvex lens developed with a flow rate ratio of 13 . We measured the intensity profiles of the beam before (C), at (A), and after (B) the focal point. The beam widths are defined as the full width at half maximum of each fitting Gaussian curve and measured as 185, 52, and $74 \mu \mathrm{m}$ at line $\mathrm{C}, \mathrm{A}$, and B, respectively. The solid lines denote the experimental results, and the discrete marks represent the fitting Gaussian curves

Fig. 7 Relationship between focal length of the optofluidic biconvex lens and flow rate ratio $\left(Q_{\text {core }} / Q_{\text {cladding }}\right)$. In the measurement of focal length, the flow rates of the two cladding streams were kept at $1 \mathrm{ml} / \mathrm{h}$, and the flow rate of the core stream was varied from 2 to $18 \mathrm{ml} / \mathrm{h}$. By increasing the flow rate ratio, the focal length can be tuned as the result of the change of the curvature of the lens interface. The solid line in the figure represents the analytic result, the cross marks denote the results obtained from numeric simulation. The inset in the figure illustrates the axial aberration retrieved from the computation of numeric simulation

Fig. 8 a The light rays propagated without scattering when the circular chamber was fully filled with a liquid with a refractive index matched to that of PDMS ( $n=$ 1.412). b The light rays were diffusively scattered when the circular chamber was 
fully filled with cinnamaldehyde $(n=1.62)$. In both cases, bright field images and fluorescence images are shown 


\section{Flow direction}

(a)

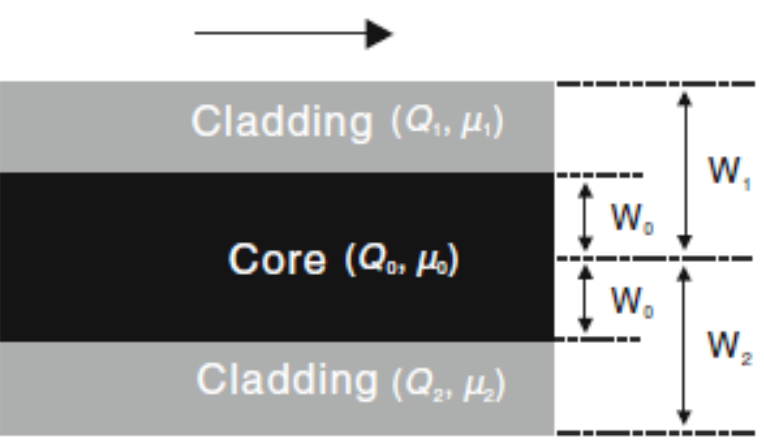

(b)

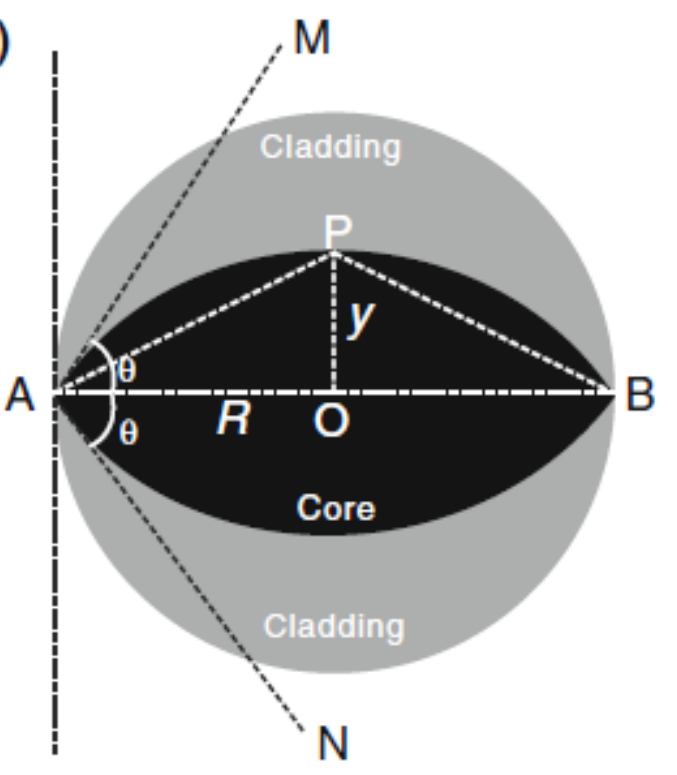

(c)

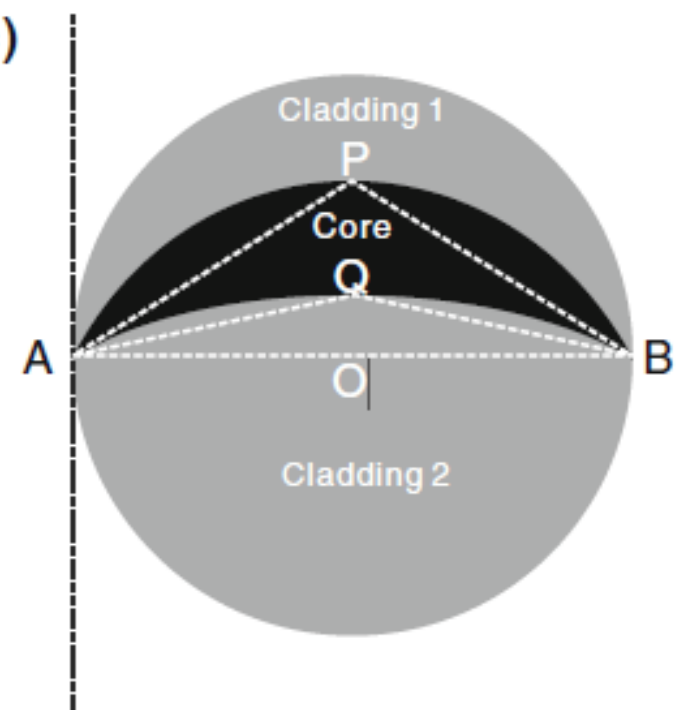

Fig. 1 


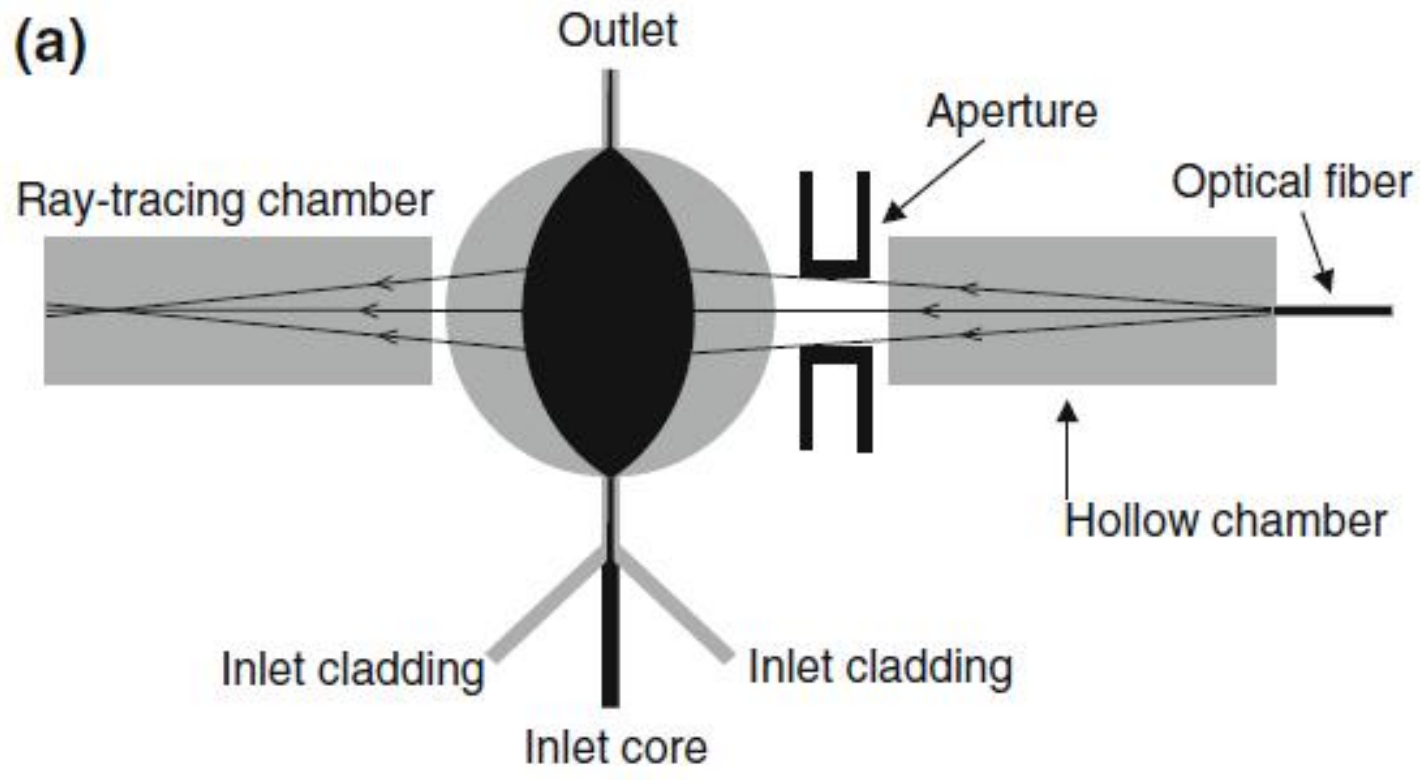

(b)

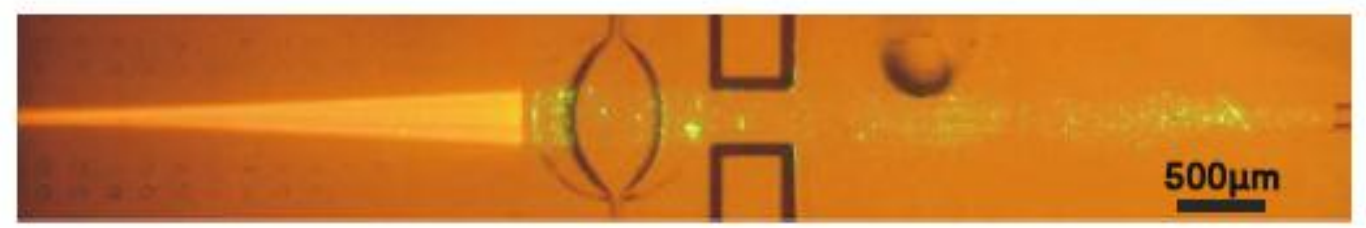

Fig. 2 


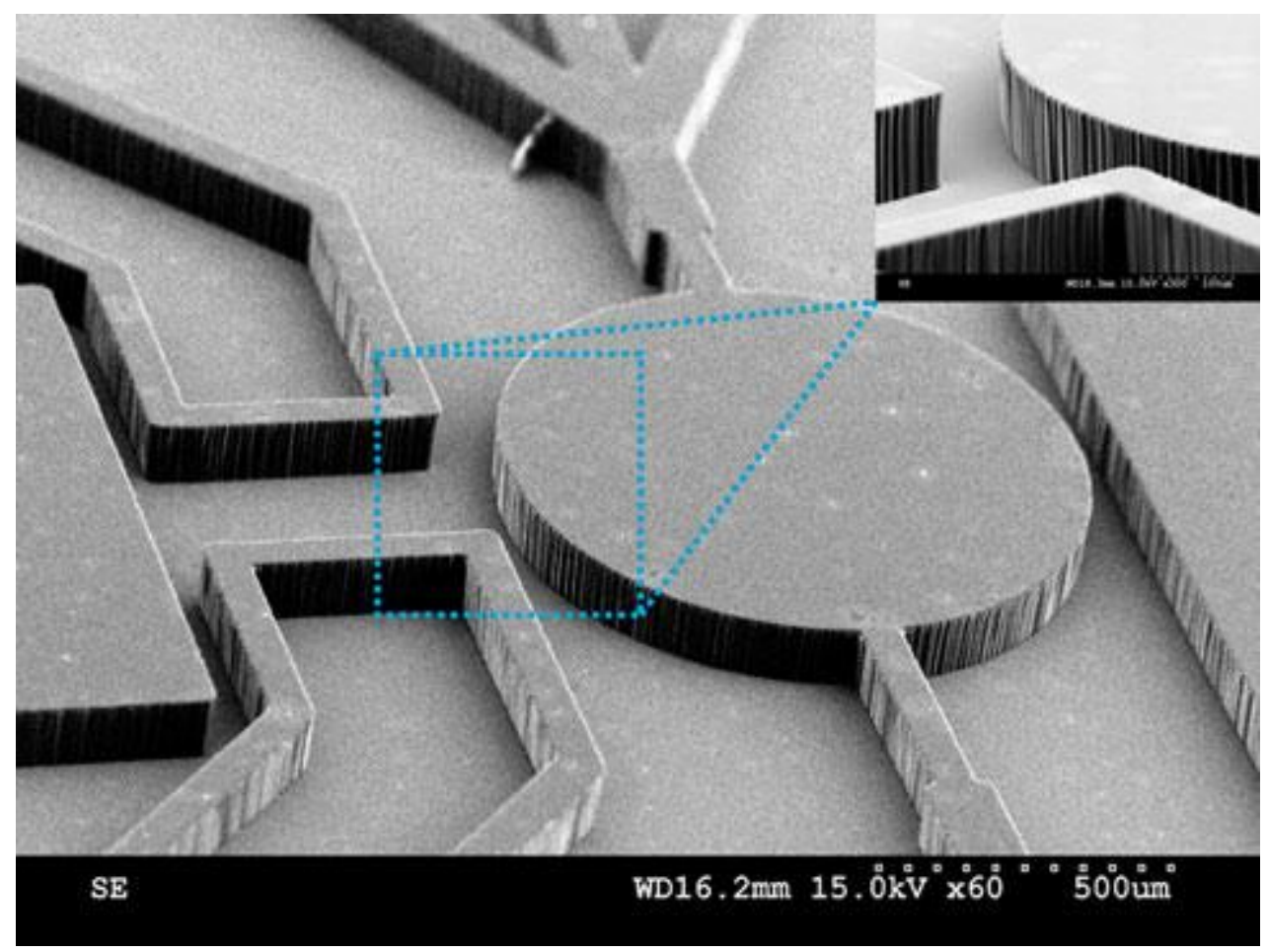

Fig. 3 


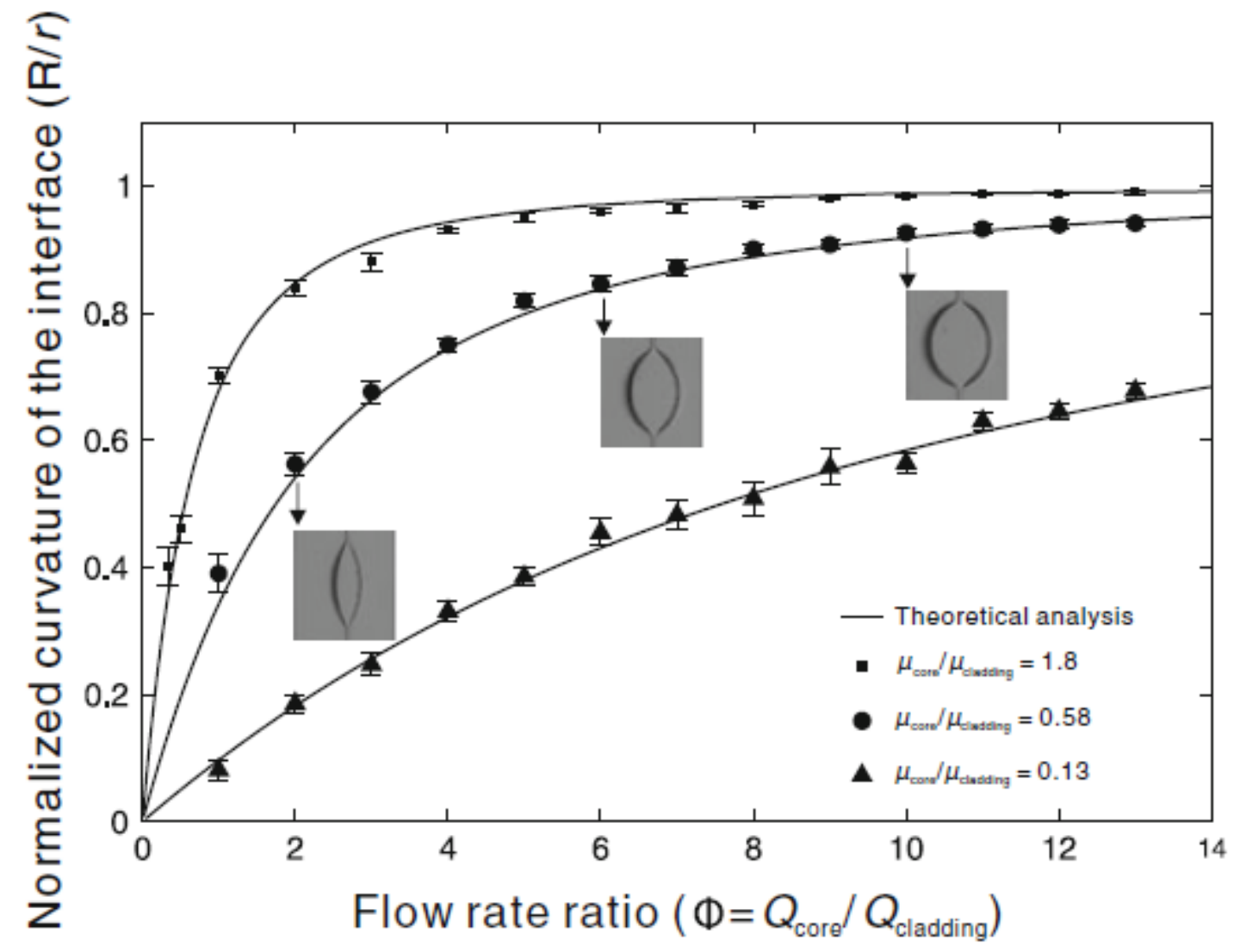

Fig. 4 
(a)
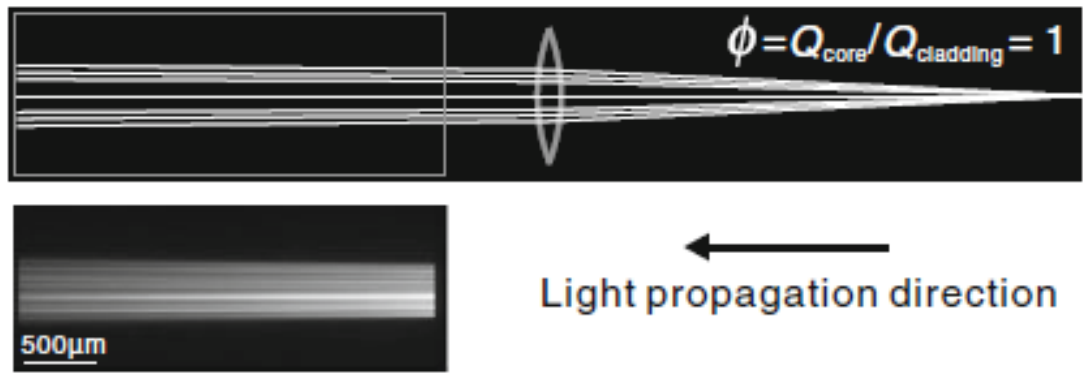

Light propagation direction

(b)
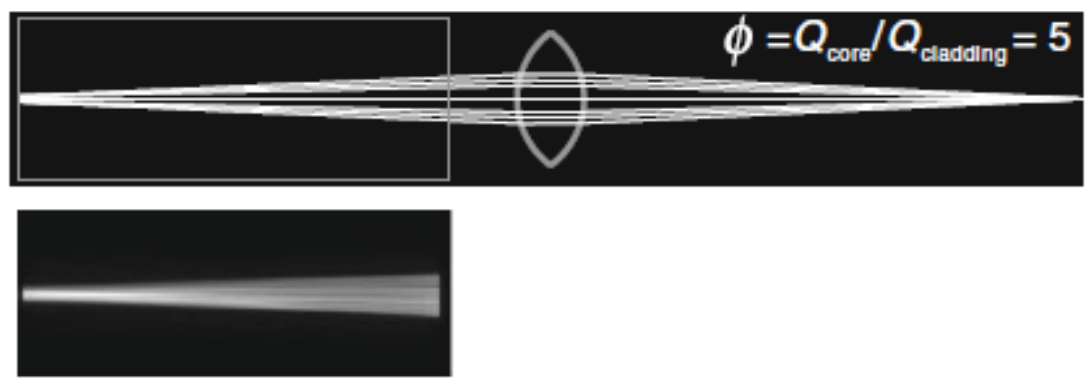

(c)
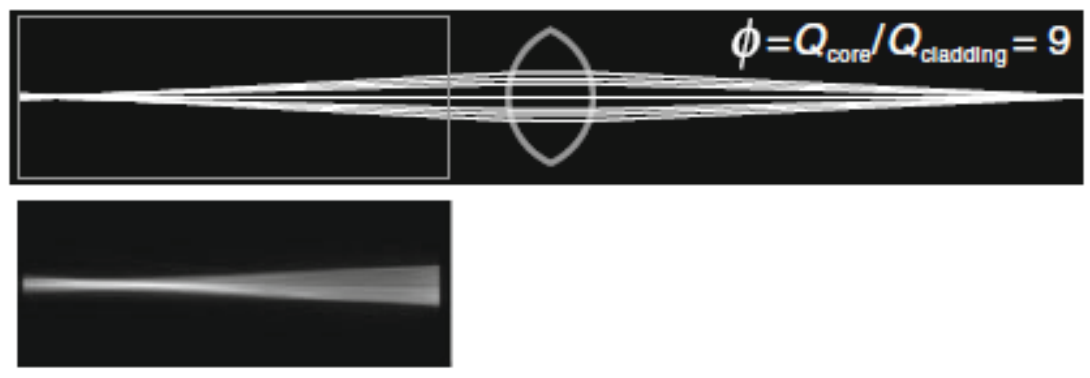

(d)

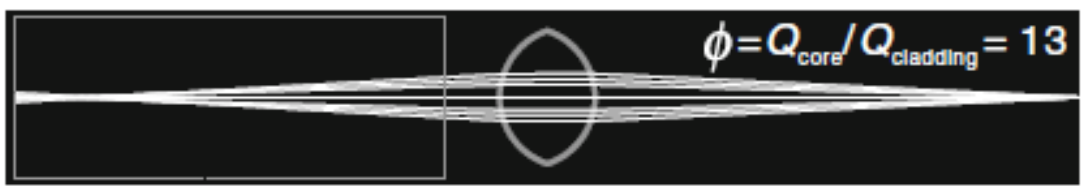

(e)
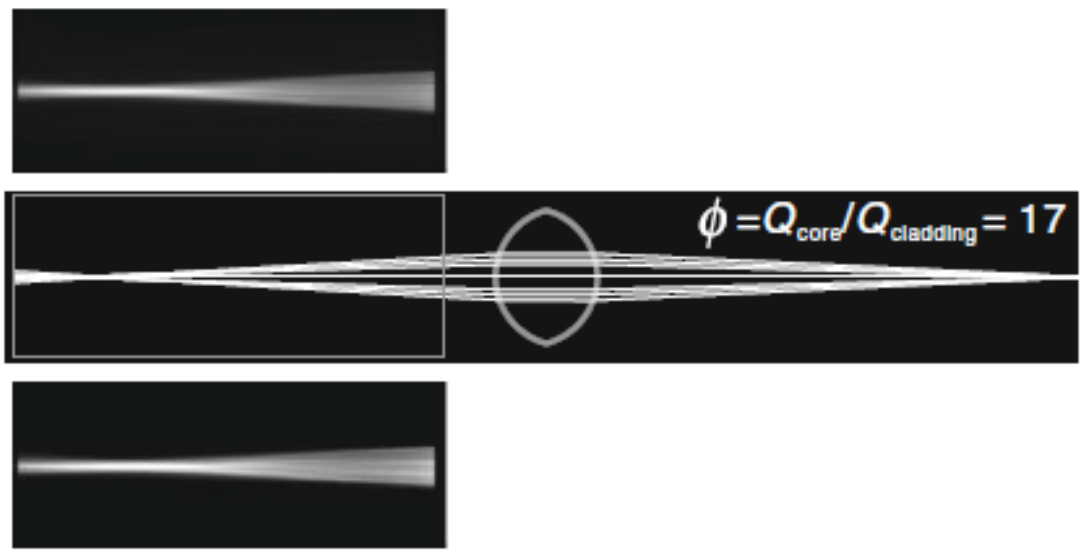

Fig. 5 
(a)

(b)
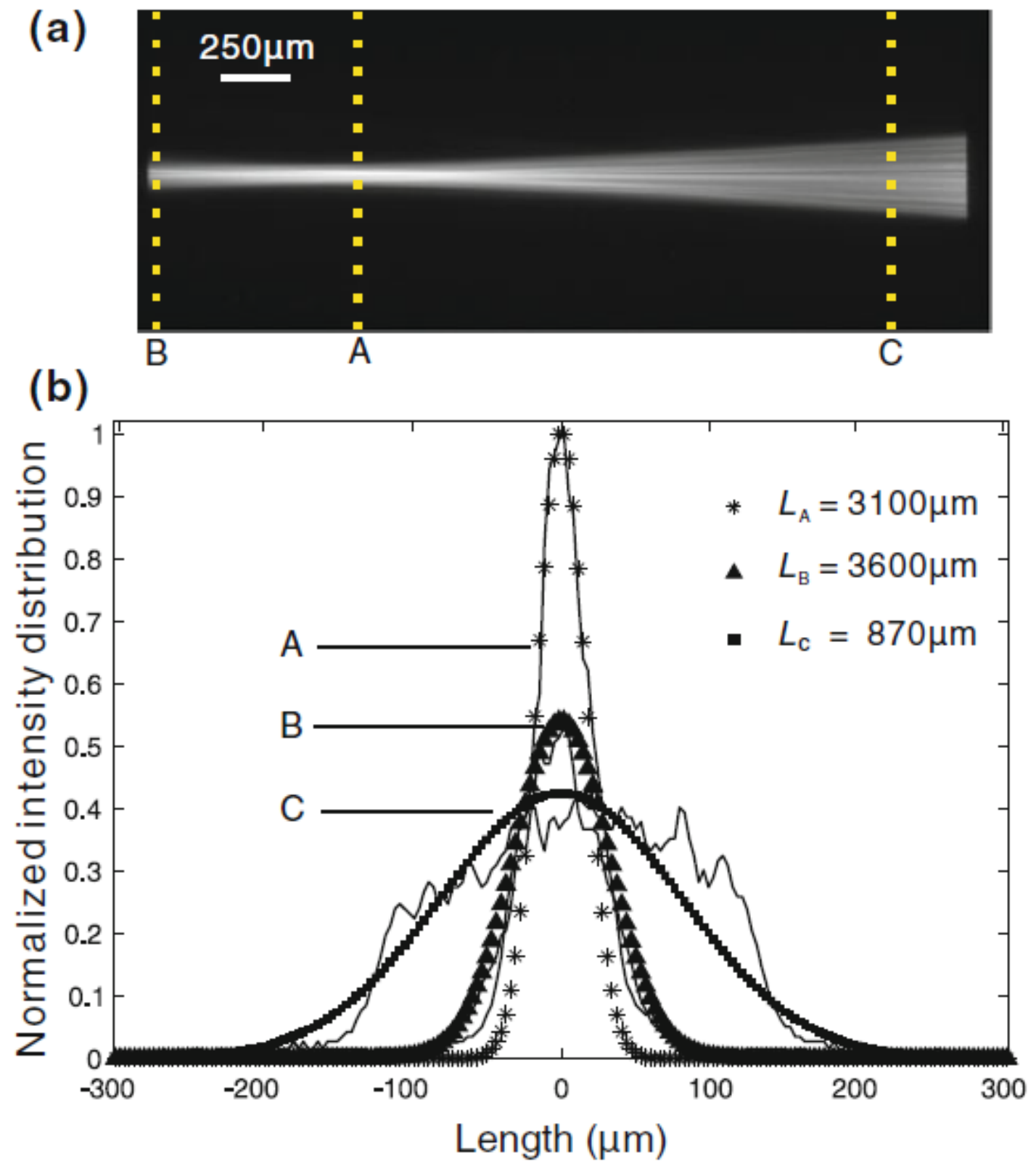

Fig. 6 


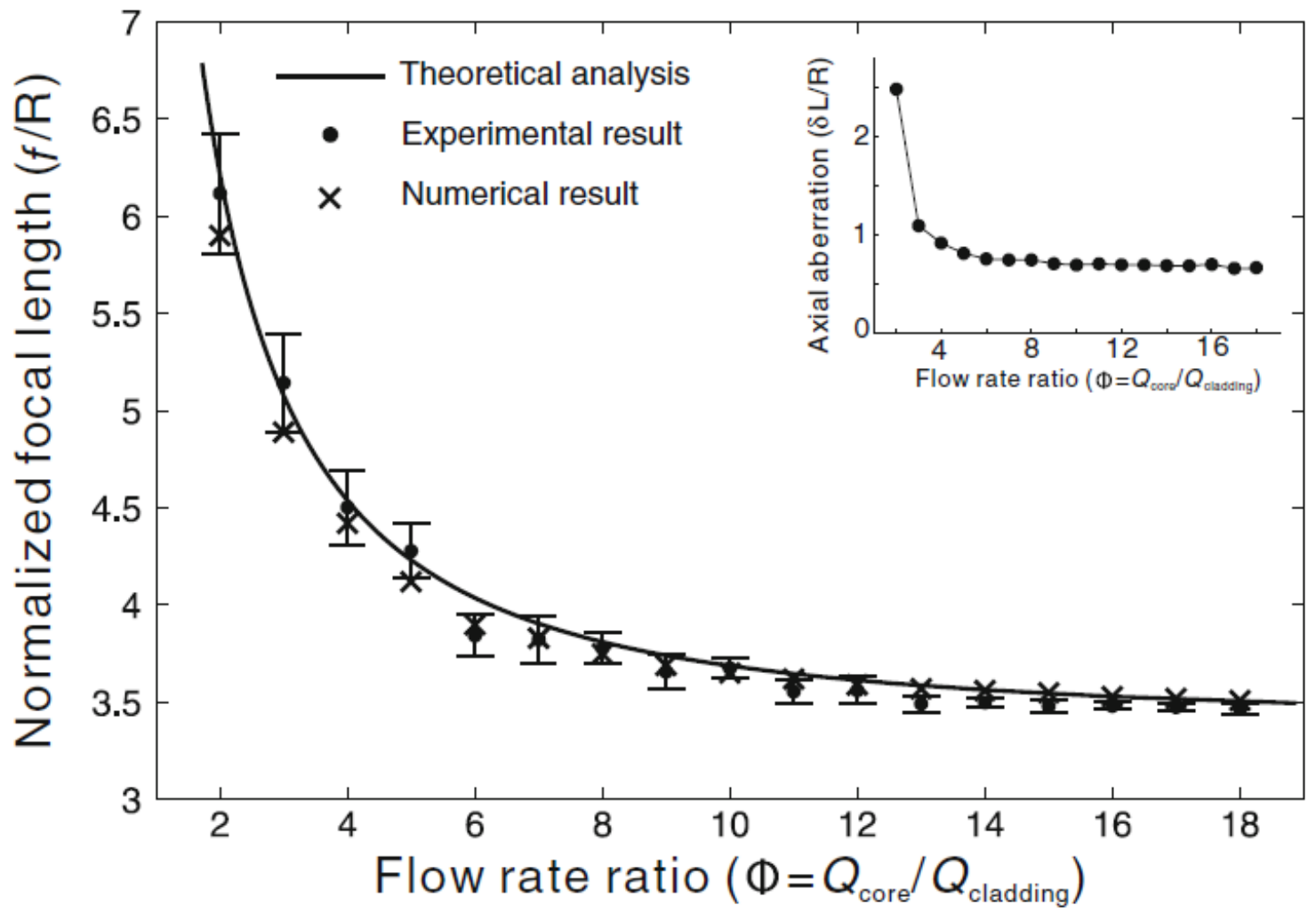

Fig. 7 
(a)

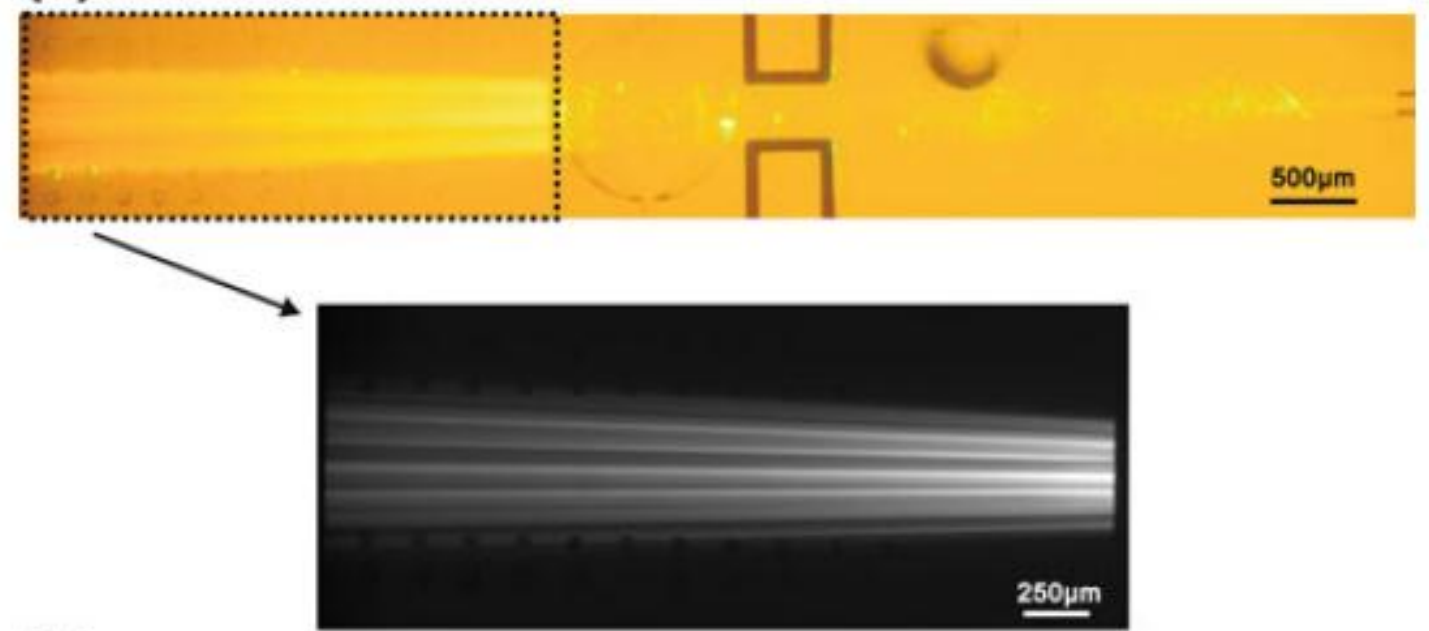

(b)

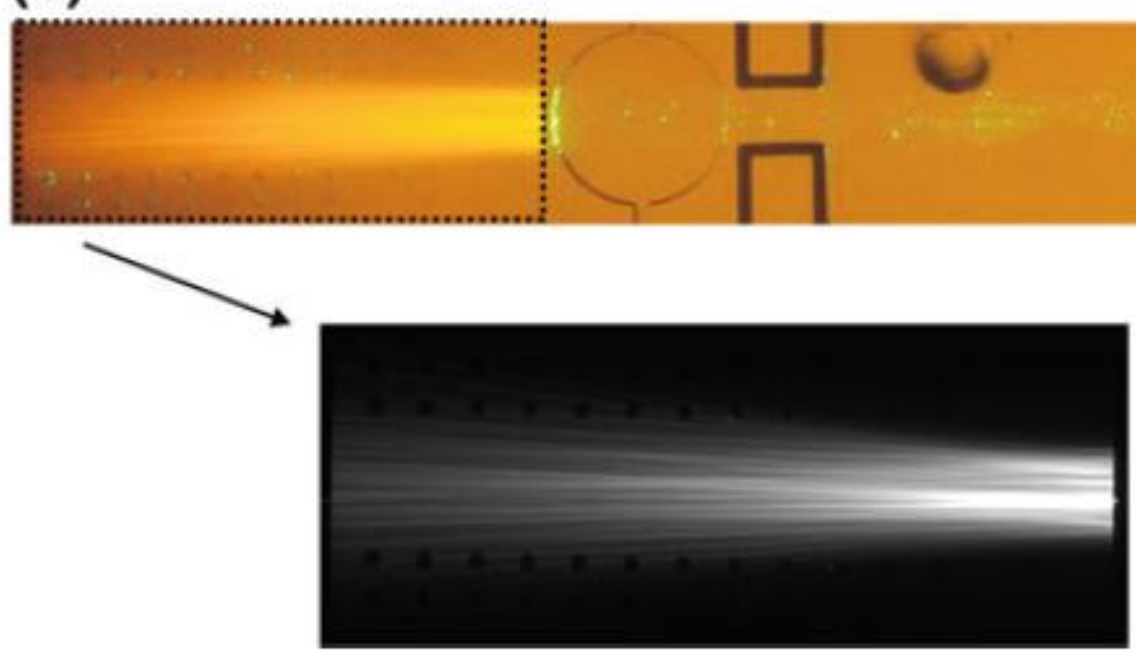

Fig. 8 